\title{
Integrin alpha 2 (Itga2), CD49b, is differentially expressed in pancreatic ductal adenocarcinoma.
}

Shahan Mamoor, MS1

1Thomas Jefferson School of Law

San Diego, CA 92901

mamoorsk@tjsl.edu

Pancreatic ductal adenocarcinoma (PDAC) is the fourth leading cause of death from cancer in the United States $(1,2)$. Novel therapies are required to extend survival in PDAC and systemslevel analysis of the tumors transcriptome as compared to the tissue of origin can reveal the basic transcriptional nature of tumors and how they differ from the tissue in which they reside and from which they originate (3). In this study, we compared the transcriptome of PDAC tumors isolated from patients with PDAC as compared to healthy, non-affected pancreatic tissue using two separate datasets $(4,5)$. We found that the cell adhesion molecule integrin alpha 2 (Itga2), also known as CD49b, was among the genes whose expression was most significantly different between PDAC and the benign pancreas. Itga2 expression was significantly higher in PDAC tumors compared to non-affected pancreatic tissue. Pancreatic ductal adenocarcinomas increase the expression of Itga2 during the transition from benign pancreatic tissue to transformed pancreatic tumor.

Keywords: PDAC, pancreatic ductal adenocarcinoma, Itga2, integrin alpha-2, tumor transcriptome analysis, systems oncology, targeted therapeutics in cancer. 


\section{Introduction}

37,000 of the 44,000 patients that were diagnosed with pancreatic ductal adenocarcinoma (PDAC) in 2012 expired from the disease (2). PDAC is expected to become the second leading cause of cancer death by 2030 (2). The current standard of care is gemcitabine which offers median survival of less than six months $(6,7)$. There are no targeted treatments or chemotherapies for PDAC; design of novel therapies for PDAC requires identification of therapeutic targets in a rationale manner. Differential gene expression analysis identifies the genes whose expression is most different between tumor and tissue of origin or tumor and metastasis in the total transcriptome and in an unbiased manner (3). Here, we compared the transcriptomes of PDAC tumors with control, non-affected pancreatic tissue using independent datasets $(4,5)$. We found that the cell adhesion molecule integrin alpha 2 (Itga2) was one of the most differentially expressed genes in the PDAC transcriptome when compared to pancreatic tissue. The expression of Itga2 was significantly higher in PDAC tumors than in the pancreas. Itga2 should be assessed for its ability to support transformation, tumor maintenance or progression.

\section{Methods}

GSE62452 was utilized for this analysis. In GSE62452, n=61 for pancreatic tissue (control) and n=69 for PDAC tumors. Affymetrix GeneChip Human Gene 1.0 ST arrays were used by the investigators who created GSE62452.

GSE62125 was also utilized for this analysis. In GSE62125, $\mathrm{n}=13$ pancreatic tissue samples (control) and $n=93$ for PDAC tumors originating from the head of the pancreas and $\mathrm{n}=25$ for PDAC tumors originating from the body and tail. Affymetrix Human Genome U219 Array was used by the investigators who created GSE62125.

The Benjamini and Hochberg method of $p$-value adjustment was utilized, and p-value adjustment was set to "Auto-detect". The NCBI generated category of platform annotation was utilized. To evaluate the statistical significance of differences in RNA expression values in 
control versus tumor groups, a two-tailed, unpaired t test with Welch's correction was performed to obtain a p-value. P-values less than 0.05 were considered statistically significant.

\section{Results}

We performed whole-transcriptome comparison of pancreatic tumors and benign pancreatic tissue to understand the most significant transcriptional differences between PDAC and the tissues of the organ from which it originates. Analysis of two separate datasets revealed that ITGA2, also known as CD49b, was among the genes whose expression changed the most significantly from pancreas to PDAC.

Pancreatic ductal adenoma carcinomas express ITGA2 significantly differently than benign pancreatic tissues do.

Itga2 was the 24th most differentially expressed gene out of 33297 total transcripts detected and measured in a sample of 61 tumors and 69 control pancreatic tissues (Table 1). This was statistically significant (Table $1 ; p=1.33 E-16) .99 .9 \%$ of the transcriptome was subject to less change in expression between control and tumor groups than Itga2 was.

Itga2 was the 162nd most differentially expressed gene out of 49386 total transcripts detected and measured in a separate dataset of 13 control pancreatic tissues and 93 PDAC tumors from the head of the pancreas. This was statistically significant (Table $1 ; p=7.59 E-29)$. When comparing the transcriptomes of the same control pancreatic tissues with the transcriptome from tumors of the body and tail of the pancreas as opposed to the head of the pancreas $(n=25)$, Itga2 was the 385th most differentially expressed gene out of 49386 total transcripts. This was statically significant but less so than in the head of the pancreas (Table 1; $p=6.91 E-15)$.

Thus, when comparing the transcriptome of tumors from patients with PDAC, either isolated from the whole organ or specifically from the head or body and tail of the organ, the 
integrin alpha 2 gene, Itga2, was significantly differentially expressed, more so than the vast majority of the genome.

Itga2 is expressed at significantly higher levels in PDAC tumors than in the pancreas.

We next obtained the exact mRNA expression values for Itga2 in each PDAC patient tumor and in each control pancreatic tissue sample to compare the expression of Itga2 in tumors versus the pancreas. We also performed a statistical test to evaluate the significance of difference in gene expression of Itga2 between PDAC tumors and the pancreas.

Itga2 was expressed at significantly higher levels in the tumors of patients diagnosed with PDAC, whether isolated from the total organ or from the head of the organ, compared to (Figure 1). For both tumors isolated from any portion of the pancreas (Figure 1; $<<0.0001$ ), or tumors isolated specifically from the head of the pancreas (Figure $1 ; p<0.0001$ ), higher expression of Itga2 in PDAC tumors was statistically significant.

Thus, we found that Itga2 was not only among the genes whose expression changes most significantly between PDAC tumor and benign pancreatic tissue, but that it was expressed at significantly higher levels in PDAC tumors than in the pancreas.

\section{Discussion}

Using independent datasets $(4,5)$, with primary tumor tissues from separate groups of human patients, and with microarray data prepared by independent investigators, we found that Itga2 was among the genes whose expression was most different between PDAC tumors and the non-affected tissue of the pancreas. The change in expression of Itga2 between tumor and benign pancreatic tissues was greater than the change in expression of $99 \%$ of the total transcriptome. Integrin alpha-2 is a cell adhesion molecule that participates in cell-cell interactions (8), possesses a binding site for collagens (9) and laminins (10), and is expressed on the surface of platelets (11). 
One previous study has showed that Itga2 mediated the acquisition of a malignant phenotype of PDAC characterized by chemoresistance (12). Another recent microarray metaanalysis identified Itga2 as up-regulated in pancreatic cancers but did not elaborate further on Itga2 in tumors versus benign tissues (13). Further, Ren et al. did not compare or address Itga2 differential expression in distinct portions of the pancreas (i.e, the head or body and tail of the pancreas) (12). Another systems-level study assessing CpG methylation in the tumors of patients with PDAC found that the Itga2 locus was hypomethylated, that this correlated with increased expression of Itga2, and that higher expression of Itga2 was associated with worse median survival (13). Together, previous literature supports the findings here that Itga2 is expressed at higher levels in the tumors of patients and argue that higher expression of Itga2 is associated with poorer survival outcomes in patients with PDAC.

Polymorphisms in Itga2 are associated with a greater risk of developing gastric cancer (14), and blocking Itga2 with an antibody lead to apoptosis and decreased migration capacity of the AGS cell line (15).

We found that Itga2 is expressed at significantly higher levels in the tumors of patients with PDAC than in the pancreas, and that Itga2 expression changed more significantly than all but 23 genes when comparing total transcription in PDAC tumors with that of the pancreas. Expression of either miR-128 (16) or miR-373 (17) has been shown to lead to decreased expression of Itga2. Enforced or over-expression of either microRNA could be a strategy to lower the expression of Itga2 in xenograft mouse models of PDAC in order to assess whether tumor formation, tumor maintenance, or tumor progression are affected by modulation of Itga2 levels in vivo $(18,19,20)$. Direct genetic inhibition by shRNA knockdown is also a possibility in xenograft models but less feasible as a therapeutic strategy for humans. An antibody targeting Itga2 has already been utilized in experimental studies in mice (21). Itga2 should be rigorously vetted as a therapeutic target in pancreatic ductal adenocarcinoma. 


\section{References}

1. Hezel, A.F., Kimmelman, A.C., Stanger, B.Z., Bardeesy, N. and DePinho, R.A., 2006. Genetics and biology of pancreatic ductal adenocarcinoma. Genes \& development, 20(10), pp.1218-1249.

2. Ying, H., Dey, P., Yao, W., Kimmelman, A.C., Draetta, G.F., Maitra, A. and DePinho, R.A., 2016. Genetics and biology of pancreatic ductal adenocarcinoma. Genes \& development, 30(4), pp.355-385.

3. Liang, P. and Pardee, A.B., 2003. Analysing differential gene expression in cancer. Nature Reviews Cancer, 3(11), p.869.

4. Yang, S., He, P., Wang, J., Schetter, A., Tang, W., Funamizu, N., Yanaga, K., Uwagawa, T., Satoskar, A.R., Gaedcke, J. and Bernhardt, M., 2016. A novel MIF signaling pathway drives the malignant character of pancreatic cancer by targeting NR3C2. Cancer research, 76(13), pp.3838-3850.

5. Janky, R.S., Binda, M.M., Allemeersch, J., Govaere, O., Swinnen, J.V., Roskams, T., Aerts, S. and Topal, B., 2016. Prognostic relevance of molecular subtypes and master regulators in pancreatic ductal adenocarcinoma. BMC cancer, 16(1), p.632.

6. King, R.S., 1996. Gemcitabine. New first-line therapy for pancreatic cancer. Cancer practice, $4(6)$, pp.353-354.

7. Burris, H.3., Moore, M.J., Andersen, J., Green, M.R., Rothenberg, M.L., Modiano, M.R., Christine Cripps, M., Portenoy, R.K., Storniolo, A.M. and Tarassoff, P., 1997. Improvements in survival and clinical benefit with gemcitabine as first-line therapy for patients with advanced pancreas cancer: a randomized trial. Journal of clinical oncology, 15(6), pp. 2403-2413.

8. Carter, W.G., Wayner, E.A., Bouchard, T.S. and Kaur, P., 1990. The role of integrins alpha 2 beta 1 and alpha 3 beta 1 in cell-cell and cell-substrate adhesion of human epidermal cells. The Journal of cell biology, 110(4), pp.1387-1404.

9. Tuckwell, D., Calderwood, D.A., Green, L.J. and Humphries, M.J., 1995. Integrin alpha 2 Idomain is a binding site for collagens. Journal of cell science, 108(4), pp.1629-1637.

10. Languino, L.R., Gehlsen, K.R., Wayner, E., Carter, W.G., Engvall, E. and Ruoslahti, E., 1989. Endothelial cells use alpha 2 beta 1 integrin as a laminin receptor. The Journal of cell biology, 109(5), pp.2455-2462.

11. Kunicki, T.J., Orchekowski, R., Annis, D. and Honda, Y., 1993. Variability of integrin alpha 2 beta 1 activity on human platelets.

12. Carnevale, I., Coppola, S., Funel, N., Danen, E.H., Schmidt, T. and Giovannetti, E., 2018. a2 $\beta 1$ integrins are potential regulators of chemoresistance through modulation of biomechanical cues in pancreatic cancer.

13. Ren, D., Zhao, J., Sun, Y., Li, D., Meng, Z., Wang, B., Fan, P., Liu, Z., Jin, X. and Wu, H., 2019. Overexpressed ITGA2 promotes malignant tumor aggression by up-regulating PD-L1 
expression through the activation of the STAT3 signaling pathway. Journal of Experimental \& Clinical Cancer Research, 38(1), pp.1-18.

14. Chen, J., Liu, N.N., Li, J.Q., Yang, L., Zeng, Y., Zhao, X.M., Xu, L.L., Luo, X., Wang, B. and Wang, X.R., 2011. Association between ITGA2 C807T polymorphism and gastric cancer risk. World journal of gastroenterology: WJG, 17(23), p.2860.

15. Chuang, Y.C., Wu, H.Y., Lin, Y.L., Tzou, S.C., Chuang, C.H., Jian, T.Y., Chen, P.R., Chang, Y.C., Lin, C.H., Huang, T.H. and Wang, C.C., 2018. Blockade of ITGA2 induces apoptosis and inhibits cell migration in gastric cancer. Biological procedures online, 20(1), p.10.

16. Zhao, X., Wu, Y. and Lv, Z., 2015. miR-128 modulates hepatocellular carcinoma by inhibition of ITGA2 and ITGA5 expression. American journal of translational research, 7(9), p.1564.

17. Ding, W., Fan, X.L., Xu, X., Huang, J.Z., Xu, S.H., Geng, Q., Li, R., Chen, D. and Yan, G.R., 2015. Epigenetic silencing of ITGA2 by MiR-373 promotes cell migration in breast cancer. PLoS One, 10(8).

18. Krisztina, K., Aune, M., James, S.S., Guido, E. and Enrique, R., 2013. Metformin inhibits the growth of human pancreatic cancer xenografts. Pancreas, 42(5), p.781.

19. Miknyoczki, S.J., Chang, H., Klein-Szanto, A., Dionne, C.A. and Ruggeri, B.A., 1999. The trk tyrosine kinase inhibitor CEP-701 (KT-5555) exhibits significant antitumor efficacy in preclinical xenograft models of human pancreatic ductal adenocarcinoma. Clinical cancer research, 5(8), pp.2205-2212.

20. Carugo, A., Genovese, G., Seth, S., Nezi, L., Rose, J.L., Bossi, D., Cicalese, A., Shah, P.K., Viale, A., Pettazzoni, P.F. and Akdemir, K.C., 2016. In vivo functional platform targeting patient-derived xenografts identifies WDR5-Myc association as a critical determinant of pancreatic cancer. Cell reports, 16(1), pp.133-147.

21. Guo, P., Moses-Gardner, A., Huang, J., Smith, E.R. and Moses, M.A., 2019. ITGA2 as a potential nanotherapeutic target for glioblastoma. Scientific reports, 9(1), pp.1-10. 


\section{Table 1}

\begin{tabular}{|c|c|c|c|c|c|c|}
\hline & ID & p-value & $\mathbf{t}$ & B & $\log F C$ & Gene \\
\hline \multicolumn{7}{|l|}{ GSE62452 } \\
\hline 24 & 8105267 & $1.33 E-16$ & 9.50 & 27.198774 & 2.14 & ITGA2 \\
\hline \multicolumn{7}{|l|}{$\begin{array}{l}\text { GSE62125 } \\
\text { (head) }\end{array}$} \\
\hline 162 & 11743617_at & 7.59E-29 & 15.395569 & 55.117425 & 3.71 & ITGA2 \\
\hline \multicolumn{7}{|l|}{$\begin{array}{l}\text { GSE62125 } \\
\text { (body and tail) }\end{array}$} \\
\hline 385 & 11743618_at & $6.91 \mathrm{E}-15$ & 12.244956 & 23.836025 & 3.4380604 & ITGA2 \\
\hline
\end{tabular}

Table 1: Pancreatic ductal adenoma carcinomas express ITGA2 significantly differently than benign pancreatic tissues do.

The rank of differential expression globally out of 49386 transcripts (GSE62125) or 33297 transcripts (GSE62435), the $p$-value with respect to global differential expression, $t$, B, the logodds of differential expression between tumor and control tissue groups, the log fold-change in expression between tumor and control pancreatic tissue, and the Gene name are shown in this chart (extracted from GEO2R). 
ITGA2

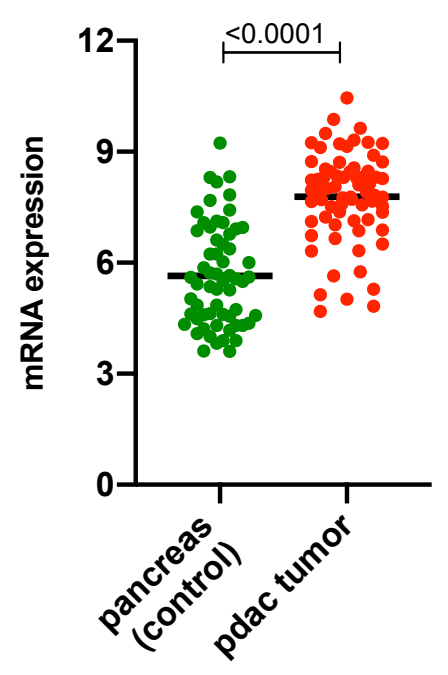

ITGA2

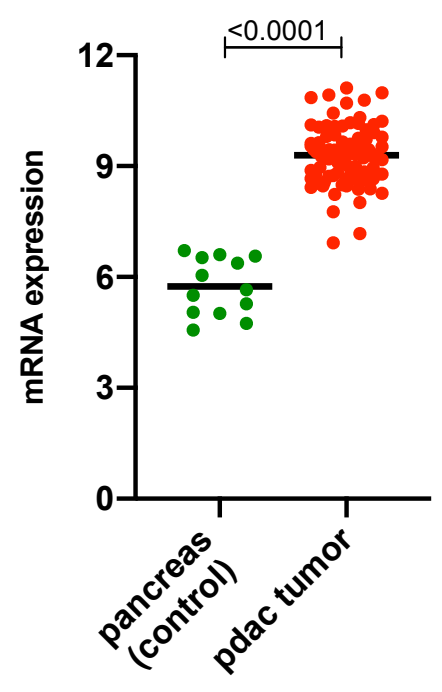

ITGA2

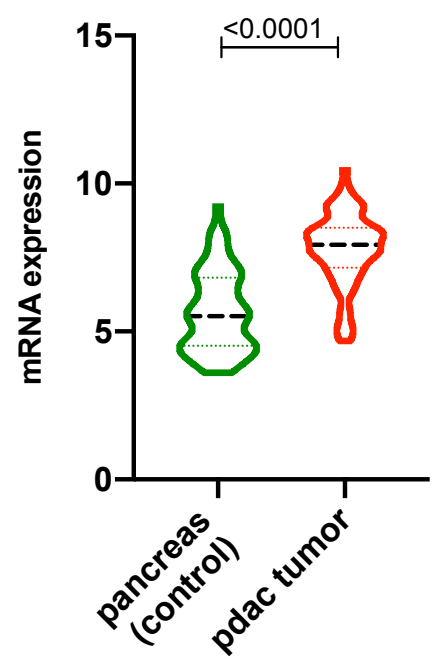

ITGA2

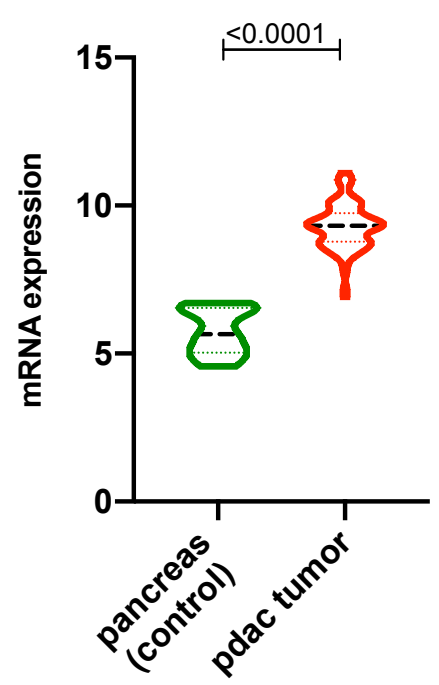

ITGA2

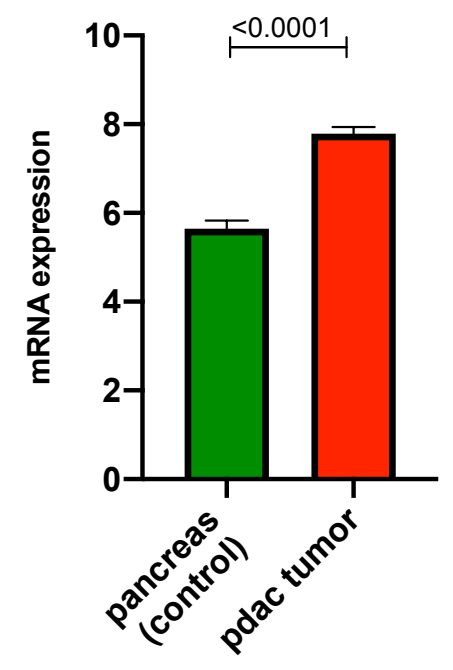

ITGA2

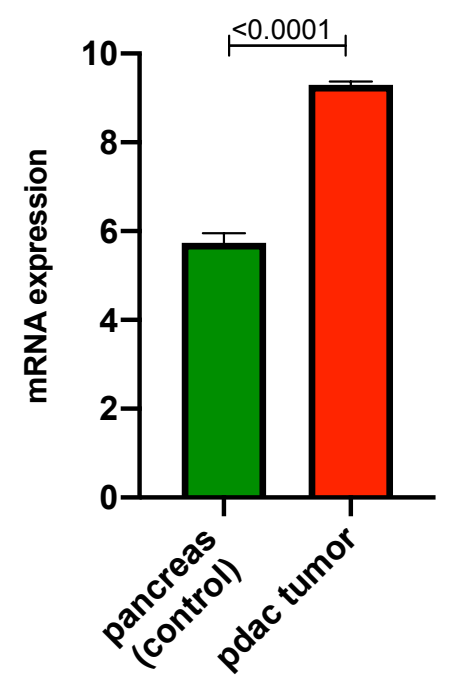

Figure 1: Itga2 is expressed at significantly higher levels in PDAC tumors than in the pancreas.

The expression of Itga2 in pancreatic ductal adenocarcinoma tumors (red) and from control, non-affected pancreatic tissue (green) from two independent datasets (GSE62452, top; GSE62125, bottom) is shown as dots denoting the expression level in each individual patient with the black horizontal line denoting the mean expression level (left); by violin plot (middle) from minimum to maximum value with the dotted black horizontal line denoting the median and the finer colored lines representing quartiles ( 25 and $75 \%$ ) (middle); as well as by bar graph with the error bars denoting the standard error of the mean (right). 